А. Д. Мижидон, А. В. Харахинов. Гибридная система дифференциальных уравнений, описывающая системы твердых тел, прикрепленных к балке Тимошенко

УДК 51-7

DOI: $10.18101 / 2304-5728-2019-1-65-77$

\title{
ГИБРИДНАЯ СИСТЕМА ДИФФЕРЕНЦИАЛЬНЫХ УРАВНЕНИЙ, ОПИСЫВАЮЩАЯ СИСТЕМЫ ТВЕРДЫХ ТЕЛ, ПРИКРЕПЛЕННЫХ К БАЛКЕ ТИМОШЕНКО
}

\author{
(C) Мижидон Арсалан Дугарович \\ доктор технических наук, профессор, \\ Восточно-Сибирский государственный университет технологий и управления \\ Россия, 670013, г. Улан-Удэ, ул. Ключевская, 40в \\ Бурятский государственный университет имени Доржи Банзарова \\ Россия, 670000, г. Улан-Удэ, ул. Смолина, 24а \\ E-mail: miarsdu@mail.ru
}

\section{(C) Харахинов Алдар Вячеславович \\ аспирант,}

Восточно-Сибирский государственный университет технологий и управления

Россия, 670013, г. Улан-Удэ, ул. Ключевская, 40в

E-mail: comma967@gmail.com

В работе для одного класса механических систем, состоящих из системы взаимосвязанных твердых тел, упруго прикрепленных к балке Тимошенко, предлагается обобщенная математическая модель, описываемая гибридной системой дифференциальных уравнений, заданной структуры. Для обобщенной математической модели разработаны теоретические основы исследования свободных колебаний, в частности, аналитико-численный метод построения частотного уравнения, основанный на рассмотрении краевой задачи для соответствующей гибридной системы дифференциальных уравнений. При этом собственные частоты по существу являются собственными значениями, при которых существует решение краевой задачи. Приведен расчетный пример, который показывает достоверность и универсальность предложенного метода исследования свободных колебаний механических систем, представляющих собой системы взаимосвязанных твердых тел, прикрепленных упругими связями к балке Тимошенко.

Ключевые слова: балка Тимошенко; краевая задача; математическая модель; твердое тело; гибридная система дифференциальных уравнений.

Для цитирования:

Мижидон А. Д., Харахинов А. В. Гибридная система дифференциальных уравнений, описывающая системы твердых тел, прикрепленных к балке Тимошенко // Вестник Бурятского государственного университета. Математика, информатика. 2019. № 1. С. 65-77.

\footnotetext{
1 Работа выполнена при финансовой поддержке РФФИ в рамках научного проекта № 18-41-030004 р_а и гранта «Молодые ученые ВСГУТУ».
} 


\section{Введение}

В статье [1] была представлена обобщенная математическая модель системы взаимосвязанных твердых тел, упруго прикрепленных к балке Эйлера - Бернулли, в виде гибридной системы дифференциальных уравнений (ГСДУ), где под ГСДУ следует понимать систему дифференциальных уравнений, состоящую как из обыкновенных дифференциальных уравнений, так и уравнений в частных производных. Обобщенная математическая модель представляет собой ГСДУ, заданной структуры, и описывает динамику различных систем взаимосвязанных твердых тел, прикрепленных упругими связями к балке Эйлера - Бернулли. Для обобщенной математической модели были разработаны теоретические основы исследования свободных колебаний. В частности, разработан аналитико-численный метод построения частотного уравнения, основанный на рассмотрении краевой задачи для соответствующей ГСДУ [2]. В [3] была исследована краевая задач для ГСДУ более общего вида. В работе [4] на основании вариационного принципа Гамильтона - Остроградского для механической системы, состоящей из балки Тимошенко с упруго прикрепленным твердым телом с двумя степенями свободы, получена математическая модель в виде ГСДУ, на основе которой в [5] произведено построение частотного уравнения. Отметим, в данном случае ГСДУ, уравнения в частных производных отличаются от уравнений в частных производных, входящих в обобщенную математическую модель, для случая балки Эйлера - Бернулли [1].

В статье в соответствии с результатами из $[1 ; 2 ; 4 ; 5]$ предлагается и исследуется обобщенная математическая модель системы взаимосвязанных твердых тел, упруго прикрепленной к балке Тимошенко в виде ГСДУ.

\section{1 Обобщенная математическая модель}

Обобщенная математическая модель для взаимосвязанных систем твердых тел, прикрепленных с помощью пружин к балке Тимошенко, в виде ГСДУ, имеет вид:

$$
\left\{\begin{array}{l}
A \ddot{z}+B z+C(D z-\bar{u})=0, \\
E I \frac{\partial^{4} u(x, t)}{\partial x^{4}}-E I \frac{\partial^{3} \beta(x, t)}{\partial x^{3}}-p I \frac{\partial^{4} u(x, t)}{\partial x^{2} \partial t^{2}}+p I \frac{\partial^{3} \beta(x, t)}{\partial x \partial t^{2}}+ \\
+p F \frac{\partial^{2} u(x, t)}{\partial t^{2}}=\sum_{i=1}^{m} q_{i}\left(d^{i T} z(t)-u(x, t)\right) \delta\left(x-a_{i}\right), \\
E I \frac{\partial^{3} u(x, t)}{\partial x^{3}}-E I \frac{\partial^{2} \beta(x, t)}{\partial x^{2}}-p I \frac{\partial^{3} u(x, t)}{\partial x \partial t^{2}}+p I \frac{\partial^{2} \beta(x, t)}{\partial t^{2}}+\chi G F \beta(x, t)=0,
\end{array}\right.
$$

где $x$ - переменная, описывающая координатную ось, совпадающую с покоящейся балкой, $a_{j}, j=\overline{1, n}-$ точки закрепления пружин, $q_{j}, j=\overline{1, n}-$ жесткость $j$-й пружины, $t-$ переменная, описывающая 
А. Д. Мижидон, А. В. Харахинов. Гибридная система дифференциальных уравнений, описывающая системы твердых тел, прикрепленных к балке Тимошенко

время, $z(t)-n$-мерная вектор-функция, описывающая смещение прикрепленных тел; $u(x, t)-$ скалярная функция; $\bar{u}-m$-мерная векторфункция с компонентами $u\left(a_{1}, t\right), u\left(a_{2},\right), \ldots, u\left(a_{m}, t\right) ; A, B$ - заданные, постоянные $n \times n$-матрицы; $C$ - заданная, постоянная $n \times m$ матрица; $D$ - заданная, постоянная $m \times n$ матрица; $d^{i}-n$-мерный вектор, составленный из строк матрицы $D,(.)^{T}-$ операция транспонирования, $\beta(x, t)$ - функция, описывающая угол сдвига, $E$ - модуль Юнга, $F-$ площадь поперечного сечения балки, $G$ - модуль сдвига балки, $\chi-$ параметр, характеризующий поперечное сечение балки, $I$ - момент инерции поперечного смещения балки, $p$ - плотность материала балки.

Отметим, что для любой расчетной схемы (в рамках некоторых допущений) произвольной системы взаимосвязанных твердых тел, прикрепленных к балке Тимошенко, ГСДУ, построенная на основании вариационного принципа Гамильтона - Остроградского, может быть представлена в виде (1).

На функции $u(x, t)$ и $\beta(x, t)$ наложены некоторые граничные условия, соответствующие условиям закрепления на концах балки:

$$
\Gamma_{1}(u(0, t), \beta(0, t))=0, \quad \Gamma_{2}(u(l, t), \beta(l, t))=0 .
$$

Решение ГСДУ (1) понимается в обобщенном смысле [6], поэтому введем понятие обобщенного решения ГСДУ, удовлетворяющей условиям (2).

Рассмотрим множество финитных вектор-функций:

$$
K=\left\{(\tilde{z}(\cdot), \tilde{u}(\cdot, \cdot), \tilde{\beta}(\cdot, \cdot))^{T}: \tilde{z}(\cdot) \in C_{\infty,[0, T]}^{n}, \tilde{u}(\cdot, \cdot) \in C_{\infty, \infty, P}, \quad \tilde{\beta}(\cdot, \cdot) \in C_{\infty, \infty, P}\right\},
$$

где $P=\left\{(x, t) \in R^{2}: 0 \leq x \leq l, 0 \leq t \leq T\right\} \quad$ - прямоугольник. Назовем основными вектор-функции из множества (3).

Определение 1. Скалярные функции $z(\cdot) \in C_{2,[o, T]}^{n}, \quad u(\cdot, \cdot) \in C_{4,2, P} \quad$ и $\beta(\cdot, \cdot) \in C_{3,2, P}$ назовем обобщенным решением краевой задачи для ГСДУ $(1)$, если функции $u(x, t)$ и $\beta(x, t)$ удовлетворяет граничным условиям краевой задачи и для любой основной вектор-функции $(\tilde{z}(\cdot), \tilde{u}(\cdot, \cdot), \tilde{\beta}(\cdot, \cdot))^{T} \in K$ справедливо 


$$
\begin{aligned}
& \int_{t_{0}}^{t_{1}}[A \ddot{z}+B z+C(D z-\bar{u})] \tilde{z}(t) d t+ \\
& +\int_{t_{0}}^{t_{0}} \int_{0}^{l}\left[E I \frac{\partial^{4} u(x, t)}{\partial x^{4}}-E I \frac{\partial^{3} \beta(x, t)}{\partial x^{3}}-\rho I \frac{\partial^{4} u(x, t)}{\partial x^{2} \partial t^{2}}+\rho I \frac{\partial^{3} \beta(x, t)}{\partial x \partial t^{2}}+\right. \\
& \left.+\rho F \frac{\partial^{2} u(x, t)}{\partial t^{2}}-\sum_{i=1}^{m} q_{i}\left(d^{i T} z(t)-u(x, t)\right) \delta\left(x-a_{i}\right)\right] \tilde{u}(x, t) d x d t+ \\
& +\int_{t_{0}}^{t_{1}} \int_{0}^{l}\left[E I \frac{\partial^{3} u(x, t)}{\partial x^{3}}-E I \frac{\partial^{2} \beta(x, t)}{\partial x^{2}}-\rho I \frac{\partial^{3} u(x, t)}{\partial x \partial t^{2}}+\right. \\
& \left.+\rho I \frac{\partial^{2} \beta(x, t)}{\partial t^{2}}+\chi G F \beta(x, t)\right] \tilde{\beta} d x d t=0 .
\end{aligned}
$$

Представив функции $z(t), \quad u(x, t), \quad \beta(x, t), \quad$ как $\quad z(t)=Z \sin \omega t$, $u(x, t)=V(x) \sin \omega t, \beta(x, t)=B(x) \sin \omega t$, из системы (1) получим алгебраическо-дифференциальную систему уравнений (5) относительно амплитудных параметров системы $Z, V(x), B(x)$ :

$$
\left\{\begin{array}{l}
\left(-\omega^{2} A+B+C D\right) Z-C \bar{V}=0, \\
\frac{d^{4} V(x)}{d x^{4}}-\frac{d^{3} B(x)}{d x^{3}}+\frac{p \omega^{2}}{E} \frac{d^{2} V(x)}{d x^{2}}-\frac{p \omega^{2}}{E} \frac{d B(x)}{d x}-\frac{p F \omega^{2}}{E I} V(x)= \\
=\sum_{i=1}^{m} q_{i}\left(d^{i T} Z-V(x)\right) \delta\left(x-a_{i}\right), \\
\frac{d^{3} V(x)}{d x^{3}}-\frac{d^{2} B(x)}{d x^{2}}+\frac{p \omega^{2}}{E} \frac{d V(x)}{d x}-\frac{p \omega^{2}}{E} B(x)+\frac{\chi G F}{E I} B(x)=0,
\end{array}\right.
$$

где $\bar{V}-m$-мерный вектор с компонентами $V\left(a_{1}\right), V\left(a_{2}\right), \ldots, V\left(a_{m}\right)$.

Граничные условия аналогичные (2) для функции $V(x)$ и $B(x)$ перепишутся в виде:

$$
\gamma_{1}(V(0), B(0))=0, \quad \gamma_{2}(V(l), B(l))=0 .
$$

Определение 2. Обобщенным решением краевой задачи системы (5)(6) будем считать вектор $Z$ и функции $V(x), B(x)$, если они удовлетворяют (5)-(6) и для любых допустимых компонентов основной векторфункции $\tilde{u}(x, t), \tilde{\beta}(x, t)$, при любом $t \in[0, T]$ справедлива система: 
А. Д. Мижидон, А. В. Харахинов. Гибридная система дифференциальных уравнений, описывающая системы твердых тел, прикрепленных к балке Тимошенко

$$
\left\{\begin{array}{l}
\int_{0}^{l}\left(\frac{d^{4} V(x)}{d x^{4}}-\frac{d^{3} B(x)}{d x^{3}}+\frac{p \omega^{2}}{E} \frac{d^{2} V(x)}{d x^{2}}-\frac{p \omega^{2}}{E} \frac{d B(x)}{d x}-\frac{p F \omega^{2}}{E I} V(x)-\right. \\
\left.-\sum_{i=1}^{m} q_{i}\left(d^{i T} Z-V(x)\right) \delta\left(x-a_{i}\right)\right) \tilde{u}(x, t) d x=0, \\
\int_{0}^{l}\left(\frac{d^{3} V(x)}{d x^{3}}-\frac{d^{2} B(x)}{d x^{2}}+\frac{p \omega^{2}}{E} \frac{d V(x)}{d x}-\frac{p \omega^{2}}{E} B(x)+\frac{\chi G F}{E I} B(x)\right) \tilde{\beta}(x, t) d x=0 .
\end{array}\right.
$$

Теорема 1. Если вектор $Z$, функции $V(x)$ и $B(x)$ удовлетворяют (5)(6), то для них справедливо:

$$
\left\{\begin{array}{l}
V(x)=\sum_{i=1}^{m} G_{i}\left(x-a_{i}\right) q_{i}\left(d^{i T} Z-V\left(a_{i}\right)\right), \\
B(x)=\sum_{i=1}^{m} B_{i}\left(x-a_{i}\right) q_{i}\left(d^{i T} Z-V\left(a_{i}\right)\right),
\end{array}\right.
$$

где функции $G_{i}(x), B_{i}(x),(i=1,2, \ldots, m)$ - это обобщенные решения системы

$$
\left\{\begin{array}{c}
\frac{d^{4} G_{i}(x)}{d x^{4}}-\frac{d^{3} B_{i}(x)}{d x^{3}}+\frac{p \omega^{2}}{E} \frac{d^{2} G_{i}(x)}{d x^{2}}-\frac{p \omega^{2}}{E} \frac{d B_{i}(x)}{d x}-\frac{p F \omega^{2}}{E I} G_{i}(x)=\delta(x) \\
\frac{d^{3} G_{i}(x)}{d x^{3}}-\frac{d^{2} B_{i}(x)}{d x^{2}}+\frac{p \omega^{2}}{E} \frac{d G_{i}(x)}{d x}-\frac{p \omega^{2}}{E} B_{i}(x)+\frac{\chi G F}{E I} B_{i}(x)=0
\end{array}\right.
$$

с краевыми условиями

$$
\gamma_{1}\left(G_{i}\left(-a_{i}\right), B_{i}\left(-a_{i}\right)\right)=0, \quad \gamma_{2}\left(G_{i}\left(l-a_{i}\right), B_{i}\left(l-a_{i}\right)\right)=0 .
$$

Доказательство. Если функции $V(x)$ и $B(x)$ удовлетворяют системе $(8)$, тогда выполнение краевых условий (6) для функций $V_{i}(x), B_{i}(x)$, $(i=1,2, \ldots, m)$ следует из краевых условий (10), вследствие линейности выражений из (8) и краевых условий (10).

Если вектор $Z$, функции $V(x)$ и $B(x)$ удовлетворяют (5), тогда они удовлетворяют (7), для любых допустимых компонент основной векторфункции $\tilde{u}(x, t), \tilde{\beta}(x, t)$. Также из первого выражения системы (7) следуeT

$$
\begin{aligned}
& \int_{0}^{l}\left(\frac{d^{4} V(x)}{d x^{4}}-\frac{d^{3} B(x)}{d x^{3}}+\frac{p \omega^{2}}{E} \frac{d^{2} V(x)}{d x^{2}}-\frac{p \omega^{2}}{E} \frac{d B(x)}{d x}-\frac{p F \omega^{2}}{E I} V(x)\right) \tilde{u}(x, t) d x= \\
& =\sum_{i=1}^{m} q_{i}\left(d^{i T} Z-V\left(a_{i}\right)\right) \tilde{u}\left(a_{i}, t\right) .
\end{aligned}
$$

Используя (8), функции $V(x)$ и $B(x)$ можно представить как:

$$
\left.V(x)=\int_{0}^{i} \sum_{i=1}^{m} G_{i}(x-\xi) q_{i}\left(d^{i T} Z-V(\xi)\right)\right) \delta\left(\xi-a_{i}\right) d \xi
$$

и 


$$
B(x)=\int_{0}^{i} \sum_{i=1}^{m} B_{i}(x-\xi) q_{i}\left(d^{i T} Z-V(\xi)\right) \delta\left(\xi-a_{i}\right) d \xi .
$$

В справедливости (8) можно убедиться с помощью подстановки (12) и (13) в левую часть (19) и во второе выражение системы (15).

Подставив (12) и (13) в левую часть (11), меняя порядок интегрирования и используя (9), получим

$$
\begin{aligned}
& \int_{0}^{l}\left(\frac{d^{4} V(x)}{d x^{4}}-\frac{d^{3} B(x)}{d x^{3}}+\frac{p \omega^{2}}{E} \frac{d^{2} V(x)}{d x^{2}}-\frac{p \omega^{2}}{E} \frac{d B(x)}{d x}-\frac{p F \omega^{2}}{E I} V(x)\right) \tilde{u}(x, t) d x= \\
& =\int_{0}^{l} \int_{0}^{l}\left(\sum _ { i = 1 } ^ { m } \left(\frac{d^{4} G_{i}(x-\xi)}{d x^{4}}-\frac{d^{3} B_{i}(x-\xi)}{d x^{3}}+\frac{p \omega^{2}}{E} \frac{d^{2} G_{i}(x-\xi)}{d x^{2}}-\frac{p \omega^{2}}{E} \frac{d B_{i}(x-\xi)}{d x}-\right.\right. \\
& \left.-\frac{p F \omega^{2}}{E I} G_{i}(x-\xi)\right) q_{i}\left(d^{i T} Z-V(\xi)\right) \delta\left(\xi-a_{i}\right) \tilde{u}(x, t) d x d \xi= \\
& =\int_{0}^{l} \sum_{i=1}^{m} \int_{0}^{i} q_{i}\left(d^{i T} Z-V(\xi) \tilde{u}(x, t) \delta(x-\xi) \delta\left(\xi-a_{i}\right) d x d \xi\right)= \\
& =\int_{0}^{i} \sum_{i=1}^{m} q_{i}\left(d^{i T} Z-V(\xi)\right) \tilde{u}(\xi, t) \delta\left(\xi-a_{i}\right) d \xi=\sum_{i=1}^{m} q_{i}\left(d^{i T} Z-V\left(a_{i}\right)\right) \tilde{u}\left(a_{i}, t\right),
\end{aligned}
$$

что равно правой части (11).

Аналогичной подставкой во второе выражение из (7) показывается выполнение равенства. Теорема доказана.

\section{2 Частотное уравнение}

Определим условия, при которых существует решение алгебраическодифференциальной системы уравнений (5) с краевыми условиями (6).

Исключим из системы (9) переменные $B_{i}(x)$. Для этого продифференцируем второе уравнение из (9) и отнимем от него первое уравнение системы (9). Полученное соотношение:

$$
\frac{d B_{i}(x)}{d x}=-\frac{p \omega^{2}}{\chi G} G_{i}(x)-\frac{\delta(x)}{\chi G F}
$$

подставим в первое уравнение системы (9). В результате получим

$$
\begin{aligned}
& \frac{d^{4} G_{i}(x)}{d x^{4}}+\left(\frac{p \omega^{2}}{\chi G}+\frac{p \omega^{2}}{E}\right) \frac{d^{2} G_{i}(x)}{d x^{2}}+\left(\frac{p^{2} \omega^{4}}{\chi G E}-\frac{p F \omega^{2}}{E I}\right) G_{i}(x)= \\
& =\left(1-\frac{p \omega^{2}}{\chi G F E}\right) \delta(x)-\frac{1}{\chi G F} \frac{d^{2} \delta(x)}{d x^{2}} .
\end{aligned}
$$

Заметим решение уравнения (14) понимается в обобщенном смысле. Если $G_{i}(x)$ некоторое обобщенное решение уравнения (14), то при любой функции $\varphi(x)$ из некоторого класса основных функций [6] должно выполняться тождество: 
А. Д. Мижидон, А. В. Харахинов. Гибридная система дифференциальных уравнений, описывающая системы твердых тел, прикрепленных к балке Тимошенко

$$
\begin{aligned}
& \int_{0}^{l}\left(\frac{d^{4} G_{i}(x)}{d x^{4}}+\left(\frac{p \omega^{2}}{\chi G}+\frac{p \omega^{2}}{E}\right) \frac{d^{2} G_{i}(x)}{d x^{2}}+\left(\frac{p^{2} \omega^{4}}{\chi G E}-\frac{p F \omega^{2}}{E I}\right) G_{i}(x)\right) \varphi(x) d x= \\
& =\int_{0}^{l}\left(\left(1-\frac{p \omega^{2}}{\chi G F E}\right) \delta(x)-\frac{1}{\chi G F} \frac{d^{2} \delta(x)}{d x^{2}}\right) \varphi(x) d x .
\end{aligned}
$$

Используя правило дифференцирования обобщенных функций, представим последнее выражение в виде:

$$
\begin{aligned}
& \int_{0}^{l}\left(\frac{d^{4} G_{i}(x)}{d x^{4}}+\left(\frac{p \omega^{2}}{\chi G}+\frac{p \omega^{2}}{E}\right) \frac{d^{2} G_{i}(x)}{d x^{2}}+\left(\frac{p^{2} \omega^{4}}{\chi G E}-\frac{p F \omega^{2}}{E I}\right) G_{i}(x)\right) \varphi(x) d x= \\
& =\left(1-\frac{p \omega^{2}}{\chi G F E}\right) \varphi(0)-\frac{1}{\chi G F} \frac{d^{2} \varphi(0)}{d x^{2}} .
\end{aligned}
$$

Таким образом, в дальнейшем будем понимать для обобщенного решения уравнения (14) выполнение тождества (15).

Теорема 2. Обобщенное решение уравнения (14) определяется выражением:

$$
G_{i}(x)=\left(1-\frac{p \omega^{2}}{\chi G F E}\right) \bar{G}_{i}(x)-\frac{1}{\chi G F} \frac{d^{2} \bar{G}_{i}(x)}{d x^{2}},
$$

где функции $\bar{G}_{i}(x),(i=1,2, \ldots, m)$ являются обобщенным решением уравнения

$$
\frac{d^{4} \bar{G}_{i}(x)}{d x^{4}}+\left(\frac{p \omega^{2}}{\chi G}+\frac{p \omega^{2}}{E}\right) \frac{d^{2} \bar{G}_{i}(x)}{d x^{2}}+\left(\frac{p^{2} \omega^{4}}{\chi G E}-\frac{p F \omega^{2}}{E I}\right) \bar{G}_{i}(x)=\delta(x) .
$$

Доказательство. Подставив (16) в левую часть выражения (15), после преобразований получим

$$
\begin{aligned}
& \int_{0}^{l}\left(\frac{d^{4} G_{i}(x)}{d x^{4}}+\left(\frac{p \omega^{2}}{\chi G}+\frac{p \omega^{2}}{E}\right) \frac{d^{2} G_{i}(x)}{d x^{2}}+\left(\frac{p^{2} \omega^{4}}{\chi G E}-\frac{p F \omega^{2}}{E I}\right) G_{i}(x)\right) \varphi(x) d x= \\
& =\int_{0}^{l}\left(1-\frac{p \omega^{2}}{\chi G F E}\right)\left(\frac{d^{4} \bar{G}_{i}(x)}{d x^{4}}+\left(\frac{p \omega^{2}}{\chi G}+\frac{p \omega^{2}}{E}\right) \frac{d^{2} \bar{G}_{i}(x)}{d x^{2}}+\right. \\
& \left.+\left(\frac{p^{2} \omega^{4}}{\chi G E}-\frac{p F \omega^{2}}{E I}\right) \bar{G}_{i}(x)\right) \varphi(x) d x-\int_{0}^{l}\left(\frac { 1 } { \chi G F } \frac { d ^ { 2 } } { d x ^ { 2 } } \left(\frac{d^{4} \bar{G}_{i}(x)}{d x^{4}}+\right.\right. \\
& \left.+\left(\frac{p \omega^{2}}{\chi G}+\frac{p \omega^{2}}{E}\right) \frac{d^{2} \bar{G}_{i}(x)}{d x^{2}}+\left(\frac{p^{2} \omega^{4}}{\chi G E}-\frac{p F \omega^{2}}{E I}\right) \bar{G}_{i}(x)\right) \varphi(x) d x= \\
& =\int_{0}^{l}\left(\left(1-\frac{p \omega^{2}}{\chi G F E}\right) \delta(x) \varphi_{i}(x)-\frac{1}{\chi G F} \frac{\left.d^{2} \delta(x)\right)}{d x^{2}} \varphi_{i}(x)\right) d x= \\
& =\left(1-\frac{p \omega^{2}}{\chi G F E}\right) \varphi(0)-\frac{1}{\chi G F} \frac{d^{2} \varphi(0)}{d x^{2}},
\end{aligned}
$$


что совпадает с правой частью (15). Теорема доказана.

Отметим, решение уравнения (17) удовлетворяет некоторым краевым условиям, соответствующим краевым условиям (10) системы (9). Обсуждение нахождения обобщенного решения $\bar{G}_{i}(x),(i=1,2, \ldots, m)$ уравнения (17) при некоторых краевых условиях приведено в [5].

Подставив (16) в первое выражение из (8), получим

$$
V(x)=\sum_{i=1}^{m}\left(\left(1-\frac{p \omega^{2}}{\chi G F E}\right) \bar{G}_{i}\left(x-a_{1}\right)-\frac{1}{\chi G F} \frac{d^{2} \bar{G}_{i}\left(x-a_{1}\right)}{d x^{2}}\right) q_{i}\left(d^{i T} Z-V\left(a_{i}\right)\right) .
$$

Далее, подставив последовательно в соотношение (18) значения $x=a_{i}, \quad(i=1,2, \ldots, m)$, получим систему линейных алгебраических уравнений относительно $V\left(a_{1}\right), V\left(a_{2}\right), V\left(a_{m}\right)$

$$
\begin{aligned}
& -\left(1+\left(\left(1-\frac{p \omega^{2}}{\chi G F E}\right) \bar{G}_{i}(0)-\frac{1}{\chi G F} \frac{d^{2} \bar{G}_{i}(0)}{d x^{2}}\right) q_{j} V\left(a_{j}\right)-\right. \\
& \left.-\sum_{\substack{i=1 \\
i \neq j}}^{m}\left(\left(1-\frac{p \omega^{2}}{\chi G F E}\right) \bar{G}_{i}\left(a_{j}-a_{i}\right)-\frac{1}{\chi G F} \frac{d^{2} \bar{G}_{i}\left(a_{j}-a_{i}\right)}{d x^{2}}\right) q_{i} V\left(a_{i}\right)\right)+ \\
& +\sum_{i=1}^{m}\left(\left(1-\frac{p \omega^{2}}{\chi G F E}\right) \bar{G}_{i}\left(a_{j}-a_{i}\right)-\frac{1}{\chi G F} \frac{d^{2} \bar{G}_{i}\left(a_{j}-a_{i}\right)}{d x^{2}}\right) q_{i} d^{d^{\prime}} Z=0, \\
& (j=1, \ldots, m) .
\end{aligned}
$$

Введем обозначения

$$
\lambda_{i}(x)=\left(\left(1-\frac{p \omega^{2}}{\chi G F E}\right) \bar{G}_{i}(x)-\frac{1}{\chi G F} \frac{d^{2} \bar{G}_{i}(x)}{d x^{2}}\right), \quad(i=1,2, \ldots, m) .
$$

Используя матричные обозначения, систему (19) запишем в виде

$$
N Z-M \bar{V}=0,
$$

где $M-$ матрица размерности $m \times m$ :

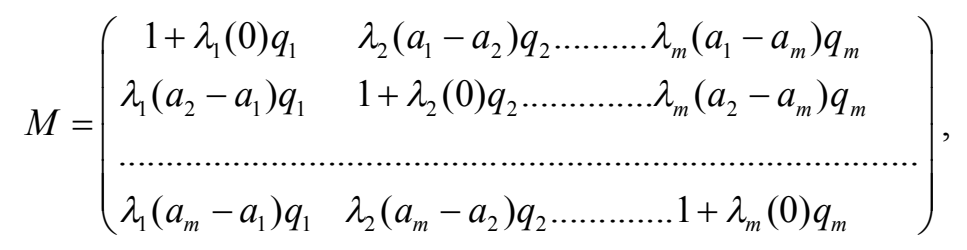

$N$ - матрица размерности $m \times n:$ 
А. Д. Мижидон, А. В. Харахинов. Гибридная система дифференциальных уравнений, описывающая системы твердых тел, прикрепленных к балке Тимошенко

$$
N=\left(\begin{array}{ll}
\sum_{i=1}^{m} \lambda_{i}\left(a_{1}-a_{i}\right) q_{i} d_{1}^{i} & \sum_{i=1}^{m} \lambda_{i}\left(a_{1}-a_{i}\right) q_{i} d_{2}^{i} \ldots \ldots \sum_{i=1}^{m} \lambda_{i}\left(a_{1}-a_{i}\right) q_{i} d_{n}^{i} \\
\sum_{i=1}^{m} \lambda_{i}\left(a_{2}-a_{i}\right) q_{i} d_{1}^{i} & \sum_{i=1}^{m} \lambda_{i}\left(a_{2}-a_{i}\right) q_{i} d_{2}^{i} \ldots \ldots \sum_{i=1}^{m} \lambda_{i}\left(a_{2}-a_{i}\right) q_{i} d_{n}^{i} \\
\ldots \ldots \ldots \ldots \ldots \ldots . . . \\
\sum_{i=1}^{m} \lambda_{i}\left(a_{m}-a_{i}\right) q_{i} d_{1}^{i} & \sum_{i=1}^{m} \lambda_{i}\left(a_{m}-a_{i}\right) q_{i} d_{2}^{i} \ldots \ldots \sum_{i=1}^{m} \lambda_{i}\left(a_{m}-a_{i}\right) q_{i} d_{n}^{i}
\end{array}\right),
$$

$\bar{V}=\left(V\left(a_{1}\right), V\left(a_{2}\right), \ldots, V\left(a_{m}\right)\right)^{T}-m$-мерный вектор.

Объединив первое уравнение системы (5) с системой (20), получим однородную систему линейных алгебраических уравнений относительно $Z, \bar{V}$.

$$
\left\{\begin{array}{l}
\left(-\omega^{2} A+B+C D\right) Z-C \bar{V}=0, \\
N Z-M \bar{V}=0 .
\end{array}\right.
$$

Однородная система алгебраических уравнений (21) имеет нетривиальные решения, в случае равенства нулю определителя. Отсюда получим уравнение собственных частот

$$
\operatorname{det}\left(\begin{array}{cc}
\left(-\omega^{2} A+B+C D\right) & -C \\
N & -M
\end{array}\right)=0 .
$$

Собственные частоты в данной постановке по существу являются собственными значениями, при которых существует решение краевой задачи (1)-(2).

\section{3 Расчетный пример}

Рассмотрим механическую систему (расчетная схема которой приведена на рис. 1), представляющую собой твердое тело, прикрепленное с помощью пружины к балке Тимошенко.

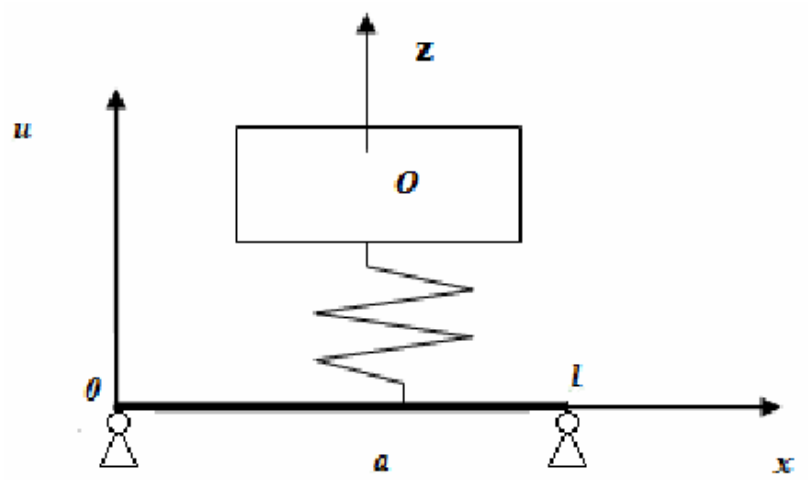

Рис. 1. Балка Тимошенко с прикрепленным упруго твердым телом 
Для расчета и сравнительного анализа были взяты данные из статьи [9]. Сравнение проводится с методом Numerical Assembly Technique (NAT). NAT - численный метод, описывающий каждый участок балки между упругими связями и проводящий их объединение с помощью граничных условий на краях каждого участка. Произведенные расчеты первых четырех собственных частот, приведены в табл. 1.

Таблица 1

Сопоставление методов

\begin{tabular}{|c|c|c|c|c|}
\hline & $\omega_{1}$ & $\omega_{2}$ & $\omega_{3}$ & $\omega_{4}$ \\
\hline NAT & 243.8150 & 643.5531 & 2513.9003 & 5573.7283 \\
\hline Предложенный метод & 243,8120 & 643.4553 & 2513.917 & 5573.627 \\
\hline
\end{tabular}

Как видно из табл. 1, результаты предложенного метода близки к данным, взятым из литературы.

\section{Заключение}

В статье для механической системы, состоящей из произвольной, взаимосвязанной системы твердых тел, прикрепленной упругими связями к балке Тимошенко, предложена обобщенная математическая модель в виде ГСДУ. Обобщенная математическая модель при некоторых допущениях относительно расчетных схем описывает широкий класс механических систем, представляющих собой системы твердых тел, прикрепленных к балке Тимошенко. В частности, в работах зарубежных авторов [713] рассматривались различные варианты систем твердых тел, прикрепленных к балке Тимошенко, которые могут быть описаны, предложенной обобщенной математической моделью (1). Для исследования свободных колебаний в приведенных работах каждый раз разрабатываются специальные, ориентированные для этих целей методы или используется метод конечных элементов. Приведенный расчетный пример показывает достоверность и универсальность изложенного в статье подхода к исследованию свободных колебаний механических систем, представляющих собой системы взаимосвязанных твердых тел, прикрепленных упругими связями к балке Тимошенко.

\section{Литература}

1. Мижидон А. Д., Дабаева М. Ж. (Цыцыренова М. Ж.) Обобщенная математическая модель системы твердых тел, установленных на упругом стержне // Вестник ВСГТУ. 2013. № 6. С. 5-12.

2. Мижидон А. Д., Баргуев С. Г. Краевая задача для одной гибридной системы дифференциальных уравнений // Вестник Бурятского государственного университета. 2013. Вып. 9. Математика и информатика. С. 130-137.

3. Мижидон А. Д., Мижидон К. А. Собственные значения для одной системы гибридных дифференциальных уравнений // Сибирские электронные математические известия. 2016. Т. 13. С. 911-922.

4. Мижидон А. Д., Харахинов А. В. К исследованию краевой задачи для бал- 
А. Д. Мижидон, А. В. Харахинов. Гибридная система дифференциальных уравнений, описывающая системы твердых тел, прикрепленных к балке Тимошенко

ки Тимошенко с упруго прикрепленным твердым телом // Вестник Бурятского государственного университета. Математика, информатика. 2016. № 1. С. 88-101.

5. Мижидон А. Д., Харахинов А. В. Частотное уравнение для балки Тимошенко с упруго прикрепленным телом с двумя степенями свободы // Вестник Бурятского государственного университета. Математика, информатика. 2016. № 4. C. 61-68.

6. Владимиров В. С. Обобщенные функции в математической физике. М.: Наука, 1976. 280 с.

7. Kukla S. Application of Green functions in frequency analysis of Timoshenko beams with oscillators // Journal of Sound and Vibration. 1997. Vol. 205, I. 3. P. 355363.

8. Majkut L. Free and forced vibration of Timoshenko beams described by single difference equation // Journal of theoretical and applied mechanics. 2009. Vol. 47, I. 1. P. 193-210.

9. Yesilce Y. Free and forced vibrations of an axially-loaded Timoshenko multispan beam carrying a number of various concentrated elements // Shock and Vibration. 2012. N. 19. P. 735-752.

10. Yesilce Y. Differential transform method and numerical assembly technique for free vibration analysis of the axial-loaded Timoshenko multiple-step beam carrying a number of intermediate lumped masses and rotary inertias // Structural Engineering and Mechanics, 2015. Vol. 53, N. 3. P. 537-573.

11. Wu J. S., Chen D. W. Free vibration analysis of a Timoshenko beam carrying multiple spring-mass systems by using the numerical assembly technique. 2001. Vol. 50, Issue 5. P. 1039-1058.

12. Magrab E. B. Natural Frequencies and Mode Shapes of Timoshenko Beams with Attachments // Journal of Vibration and Control. 2007. Vol. 13, I. 7. P. 905-934.

13. Xu S., Wang X. The discrete singular convolution for analyses of elastic wave propagations in one-dimensional structures // Applied Mathematical Modeling. 2010. Vol. 34, I. 11. P. 3493-3508.

\section{HYBRID SYSTEM OF DIFFERENTIAL EQUATIONS DESCRIBING SYSTEMS OF SOLIDS ATTACHED TO A TIMOSHENKO BEAM}

\section{Arsalan D. Mizhidon}

Dr. Sci. (Engineering), Prof.,

East-Siberian State University of Technology and Management

40v Klyuchevskaya St., Ulan-Ude 670013, Russia

Dorzhi Banzarov Buryat State University

24a Smolina St., Ulan-Ude 670000, Russia

E-mail:miarsdu@mail.ru

Aldar V. Kharakhinov

Research Assistant,

East-Siberian State University of Technology and Management

40v Klyuchevskaya St., Ulan-Ude 670013, Russia

E-mail: comma967@gmail.com 
The article proposes a generalized mathematical model described by a hybrid system of differential equations of a prescribed structure for one class of mechanical systems consisting of a system of connected solids, elastically attached to a Timoshenko beam. Theoretical background of the study of free vibrations has been developed for a generalized mathematical model, in particular, an analytical and numerical method for constructing a frequency equation based on the consideration of a boundary value problem for the corresponding hybrid system of differential equations. In this case, natural frequencies are in fact eigenvalues for which there exists a solution to a boundary value problem. A calculated example is given that shows the reliability and versatility of the proposed method for studying free vibrations of mechanical systems, which are systems of connected solids elastically attached to a Timoshenko beam.

Keywords: Timoshenko beam; boundary value problem; mathematical model; solid; hybrid system of differential equations.

\section{References}

1. Mizhidon A. D., Dabaeva M. Zh. (Tsytsyrenova M. Zh.) Obobshchennaya matematicheskaya model sistemy tverdykh tel, ustanovlennykh na uprugom sterzhne [A Generalized Mathematical Model of the System of Solids Mounted on an Elastic Rod]. Vestnik Vostochno-Sibirskogo gosudarstvennogo tekhnicheskogo universiteta. 2013. No. 6. Pp. 5-12.

2. Mizhidon A. D., Barguev S. G. Kraevaya zadacha dlya odnoi gibridnoi sistemy differentsialnykh uravnenii [Boundary Value Problem for One Hybrid System of Differential Equations]. Vestnik Buryatskogo gosudarstvennogo universiteta. 2013. V. 9. Matematika i informatika. Pp. 130-137.

3. Mizhidon A. D., Mizhidon K. A. Sobstvennye znacheniya dlya odnoi sistemy gibridnykh differentsialnykh uravnenii [Eigenvalues for One System of Hybrid Differential Equations]. Siberian Electronic Mathematical Reports. 2016. V. 13. Pp. 911922.

4. Mizhidon A. D., Kharakhinov A. V. K issledovaniyu kraevoi zadachi dlya balki Timoshenko s uprugo prikreplennym tverdym telom [Towards the Study of a Boundary Value Problem for the Timoshenko Beam with an Elastically Attached Solid]. Vestnik Buryatskogo gosudarstvennogo universiteta. Matematika, informatika. 2016. No. 1. Pp. 88-101.

5. Mizhidon A. D., Kharakhinov A. V. Chastotnoe uravnenie dlya balki Timoshenko s uprugo prikreplennym telom s dvumya stepenyami svobody [Frequency Equation for the Timoshenko beam with an Elastically Attached Body with Two Degrees of Freedom]. Vestnik Buryatskogo gosudarstvennogo universiteta. Matematika, informatika. 2016. No. 4. Pp. 61-68.

6. Vladimirov V. S. Obobshchennye funktsii v matematicheskoi fizike [Generalized Functions in Mathematical Physics]. Moscow: Nauka Publ., 1976. 280 p.

7. Kukla S. Application of Green Functions in Frequency Analysis of Timoshenko Beams with Oscillators. Journal of Sound and Vibration. 1997. V. 205. Iss. 3. Pp. 355363.

8. Majkut L. Free and Forced Vibration of Timoshenko Beams Described by Single Difference Equation. Journal of Theoretical and Applied Mechanics. 2009. V. 47. Iss. 1. Pp. 193-210.

9. Yesilce Y. Free and Forced Vibrations of an Axially-Loaded Timoshenko Multi-Span Beam Carrying a Number of Various Concentrated Elements. Shock and Vibration. 2012. No.19. Pp. 735-752. 
А. Д. Мижидон, А. В. Харахинов. Гибридная система дифференциальных уравнений, описывающая системы твердых тел, прикрепленных к балке Тимошенко

10. Yesilce Y. Differential Transform Method and Numerical Assembly Technique for Free Vibration Analysis of the Axial-Loaded Timoshenko Multiple-Step Beam Carrying a Number of Intermediate Lumped Masses and Rotary Inertias. Structural Engineering and Mechanics. 2015. V. 53. No. 3. Pp. 537-573.

11. Wu J. S., Chen D. W. Free Vibration Analysis of a Timoshenko Beam Carrying Multiple Spring-Mass Systems by Using the Numerical Assembly Technique. 2001. Vol. 50. Iss. 5. Pp. 1039-1058.

12. Magrab E. B. Natural Frequencies and Mode Shapes of Timoshenko Beams with Attachments. Journal of Vibration and Control. 2007. V. 13. Iss. 7. Pp. 905-934.

13. Xu S., Wang X. The Discrete Singular Convolution for Analyses of Elastic Wave Propagations in One-Dimensional Structures. Applied Mathematical Modeling. 2010. V. 34. Iss. 11. Pp. 3493-3508. 